\title{
Flexible Transport Network Expansion via Open WDM Interfaces
}

\section{Fagertun, Anna Manolova; Skjoldstrup, Bjarke}

Published in:

2013 International Conference on Computing, Networking and Communications (ICNC)

Link to article, DOI:

10.1109/ICCNC.2013.6504114

Publication date:

2013

Link back to DTU Orbit

\section{Citation (APA):}

Fagertun, A. M., \& Skjoldstrup, B. (2013). Flexible Transport Network Expansion via Open WDM Interfaces. In 2013 International Conference on Computing, Networking and Communications (ICNC) (pp. 385-389). IEEE. https://doi.org/10.1109/ICCNC.2013.6504114

\section{General rights}

Copyright and moral rights for the publications made accessible in the public portal are retained by the authors and/or other copyright owners and it is a condition of accessing publications that users recognise and abide by the legal requirements associated with these rights.

- Users may download and print one copy of any publication from the public portal for the purpose of private study or research.

- You may not further distribute the material or use it for any profit-making activity or commercial gain

- You may freely distribute the URL identifying the publication in the public portal

If you believe that this document breaches copyright please contact us providing details, and we will remove access to the work immediately and investigate your claim 


\title{
Flexible Transport Network Expansion via Open WDM Interfaces
}

\author{
Anna Manolova Fagertun ${ }^{1}$, Bjarke Skjoldstrup ${ }^{2}$ \\ ${ }^{1}$ DTU Fotonik, Kgs. Lyngby, Denmark, e-mail: anva@fotonik.dtu.dk; ${ }^{2}$ TDC A/S, Kbenhavn, Denmark, e-mail: bjsk@tdc.dk;
}

\begin{abstract}
This paper presents a successful test-bed implementation of a multi-vendor transport network interconnection via open WDM interfaces. The concept of applying Alien Wavelengths (AWs) for network expansion was successfully illustrated via deployment of multi-domain/multi-vendor end-to-end OTN services. We evaluate the impact of AW service establishment on both native and other alien services. Our experience confirms the technical feasibility of the concept in the context of transparent network-to-network interconnection at the optical layer. Furthermore, main operational challenges are discussed.
\end{abstract}

\section{INTRODUCTION}

$\mathbf{I}$ $\mathrm{N}$ order to meet adequately the ever increasing need for transmission capacity contemporary transport networks heavily deploy next generation Wavelength Division Multiplexing (WDM) technology in their backbones. Standardization bodies have defined clear demarcation interfaces between the network providers and their clients which guarantee delivery of services with very demanding parameters (e.g., error-free service, five 9's availability). These demarcation points necessitate termination and the associated opto-electro-optical conversion of the signals at the borders of the WDM domains, a task that is performed by deploying transponders at the edges of the network. This status-quo operation has proven to be highly efficient in delivering guaranteed performance at carrier-class level. Nonetheless, a major drawback of this solution is that the network provider is bound to the equipment produced by one specific vendor. This drawback can be alleviated by deployment of open WDM interfaces, a.k.a. Alien Wavelengths (AW). A solution for cross-vendor interoperability at the optical layer has recently been requested by several network operators and has resulted in a set of initial standardization documents within ITU-T - the G.698.X series. Within the ITU-T framework, this solution is referred to as "black link" approach.

Alien wavelengths are optical signals, originated by a transponder, which the WDM system under consideration has not been specifically designed for ${ }^{1}$. Typically, these are signals generated from transponders from a different vendor, than the one supplying the WDM transport network. The technical

This work has been supported by the Danish Advanced Technology Foundation (Hjteknologifonden) through the research project "Providing new capacity services for multi-vendor optical transport networks via an open interface"

${ }^{1}$ Note that most often the system is perfectly capable of transporting the $\mathrm{AW}$, but cannot guarantee its performance feasibility of AWs has been documented in several different scenarios over the past years. In particular two application scenarios have been illustrated and evaluated: the interconnection of core IP routers equipped with long-reach pluggable transponders via a third party WDM transport network [1][3], and the interconnection of two WDM transport segments, typically within the research and education community (NRENs) [4], [5].

Apart from applying the concept for novel service delivery to clients, AWs can be a very attractive solution for transport network expansion, i.e., deploying new network segments. Being able to freely deploy network segments build from different vendors and using different types of transmission technology illustrates an extreme case of flexible network deployment. In such a scenario, AWs can be highly useful for CAPEX savings [5]. Such application of AWs has not been thoroughly discussed within the community. Thus, the focus of this paper is on network expansion via deployment of AWs. First, we present the application scenario of AW as an open WDM interface for network expansion and outline its advantages and challenges. Then, our experimental test-bed is presented where two transport domains are interconnected via open WDM interfaces. Performance measurements are taken and a successful, post-FEC error-free service delivery is documented.

\section{FLEXIBLE NETWORK EXPANSION VIA OPEN WDM INTERFACES}

Thus far, the deployment of AWs has been demonstrated in relation to either novel service delivery (i.e., at the border between the transport network and the higher layer (typically IP) client) [1]-[3] or in relation to interconnection of long-haul transport domains (i.e., at the border between two transport service providers) [4], [5]. This work focuses on a third application scenario - the case of flexible network deployment/evolution.

Network migration is a natural process for transport networks, necessitated by growth, business expansion and new customer base. Often big transport providers buy competitors which involves integration of the acquired infrastructure into the provider's own network. This leads to multi-vendor environment within the transport network provider which needs to be handled by applying very strict interfacing principles (via transponders) at the optical layer. In such a scenario, the availability of an open WDM interface could potentially 


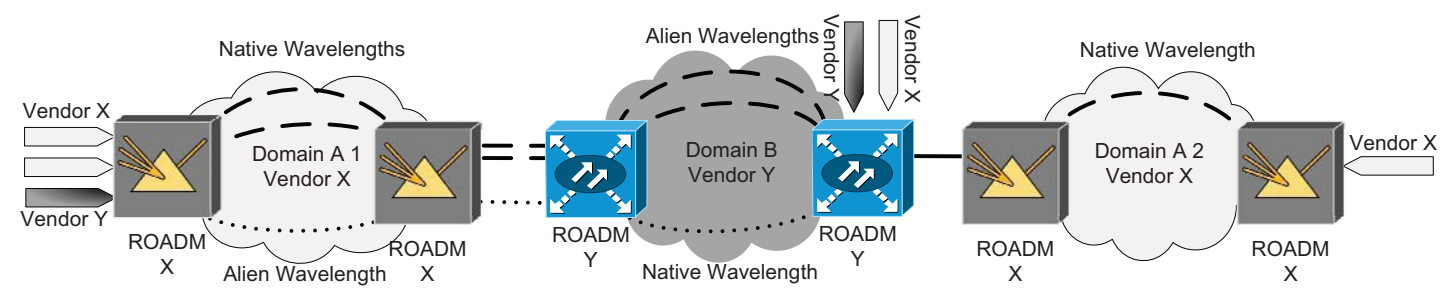

Fig. 1. Example application of Alien Wavelengths - WDM network expansion.

result in considerable financial savings [6]. One of the biggest setbacks in transport network evolution is the lack of flexibility in choosing network components freely. In practice, network operators are bound to one specific vendor, which supplies them with all needed components for their WDM infrastructure. This provides for a very high quality of the provided services, since interoperability between the components is guaranteed. On the other hand, the network provider is bound only to the functionalities the provided WDM equipment supports, since adding equipment from another vendor (e.g., for building a new network segment) necessitates interfacing via transponders at the edges of the segments, which is expensive.

Fig. 1 illustrates two applications of the AW concept within a network expansion scenario. Domain B (using WDM platform from vendor $\mathrm{Y}$ ) is being acquired by network provider A (using WDM platform from vendor $\mathrm{X}$ ). At the illustrated example provider A can use domain $\mathrm{B}$ to reach further with his native (vendor $\mathrm{X}$ ) transponders, or to interconnect two subdomains of its former network via the new domain. In the first example (interconnecting domains A1 and B) provider A will not save transponders (still the same amount of transponders is needed to serve clients), but will have the opportunity to reach further through the new network segment without OEO conversion of the signal at the border between the domains. This will reduce the processing delay, the electrical power consumption, and the sub-rack space/equipment footprint at the site on the border between the two domains. Furthermore, it will provide extended customer base since customers, previously served solely by operator B or by operator A, will be able to reach further through the new domains.

For the second expansion scenario (interconnecting domains A1 and A2 via domain B), it is clear that considerable savings in terms of deployed transponders in the network can be achieved, since there is no need for terminating the signal at the borders of domain B in order to transport client signals from domain A1 to domain A2. Thus, both scenarios benefit from the deployment of AWs.

Despite the numerous challenges related to AW deployment and operation [7], under the presented scenario some of the drawbacks can be alleviated. The lack of operational control for example can be managed since in the network expansion scenario both the WDM transport domain and the alien transponders are under the control of the same network operator. Even though there is no common network management system for control of transport equipment from different vendors, the OTN layering implemented in modern WDM systems allows for dividing the AW circuits into manageable optical trails (OCh trails) terminating at the optical interface between the systems. Thus the network management can be converted to subnetwork management in each domain and tailored to the needs of the operator, since he controls both domains. Alarms can be correlated and access to performance data is possible in an integrated management environment. This necessitates the deployment of new system for monitoring and control, which can be jointly designed and implemented in cooperation with the vendors. First steps in this direction are already available [1] though the application scenario is for the client-provider interface [2], [3].

\section{EXPERIMENTAL SETUP - CHALLENGES AND SOLUTIONS}

This paper demonstrates the interworking between two WDM transport platforms from different vendors via AWs deployment. Three types of transponders are used for endto-end service establishment. There are two novel aspects in this experimental setup. First, we mix dispersion compensated and dispersion non-compensated domains, and non-coherent and coherent transponders. Second, one of the end-to-end AWs does not have a native network segment as the AW deployments presented in previous works [4], [5]. Such a scenario poses many challenges and presents a rather extreme case of network expansion. Nevertheless, it provides us with the opportunity to evaluate more extreme operational requirements, which is useful when designing a fully open WDM layer.

\section{A. Test-bed setup}

Our experimental setup is presented on Fig. 2, and consists of two domains from vendors $\mathrm{X}$ and $\mathrm{Y}^{2}$, with corresponding $10 \mathrm{G}$ and $40 \mathrm{G}$ services as indicated. The $40 \mathrm{G}$ (coherent) signals and the $10 \mathrm{G}$ signal from vendor $\mathrm{Z}$ are used as AWs, i.e., each signal traverses both domains. The $10 \mathrm{G}$ services from vendors $\mathrm{X}$ and $\mathrm{Y}$ are native services, starting and terminating at their native domains. The domains are interconnected via direct fiber connections between the corresponding MUX/DMUX

\footnotetext{
${ }^{2}$ Due to Non-disclosure Agreements the names of the involved vendors cannot be used.
} 


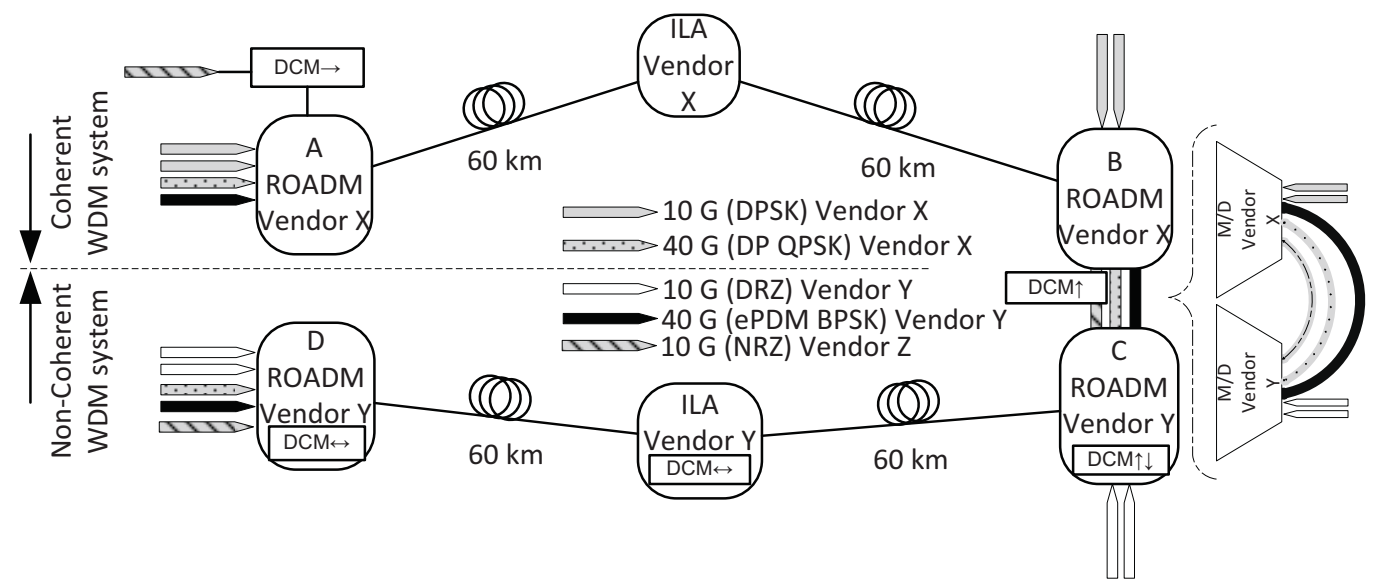

Fig. 2. Experimental test-bed: two WDM platforms and three transponder types.

ports of the ROADMS. The network segment from Vendor Y is dispersion compensated, whereas the one from $\mathrm{X}$ is not. Even though Vendor $Y$ has non-coherent system, the $40 \mathrm{G}$ transponder from the vendor is coherent, thus no dispersion compensation is needed for it while passing via the Vendor $\mathrm{X}$ 's segment of the network. The test-bed represents a metronetwork application where we expect that a greater diversity in the deployed transmission technologies (and vendors) can be seen. The channel allocation for the services is presented in Tab. I.

\begin{tabular}{|c|c|c|c|}
\hline Channel & Service & Modulation & Vendor \\
\hline $1552.52 \mathrm{~nm}$ & $10 \mathrm{G}$ & DPSK & $\mathrm{X}$ \\
$1551.72 \mathrm{~nm}$ & $10 \mathrm{G}$ & DPSK & $\mathrm{X}$ \\
$1550.92 \mathrm{~nm}$ & $40 \mathrm{G}$ & DP QPSK & $\mathrm{X}$ \\
$1550.12 \mathrm{~nm}$ & $40 \mathrm{G}$ & ePDM BPSK & $\mathrm{Y}$ \\
$1549.32 \mathrm{~nm}$ & $10 \mathrm{G}$ & DRZ & $\mathrm{Y}$ \\
$1548.52 \mathrm{~nm}$ & $10 \mathrm{G}$ & DRZ & $\mathrm{Y}$ \\
$1547.72 \mathrm{~nm}$ & $10 \mathrm{G}$ & NRZ & $\mathrm{Z}$ \\
\hline
\end{tabular}

TABLE I

ChANNel ALlocation FOR ALL SERVICES - 100 GHz SPACING.

We encountered three main challenges in the process of AW service establishment, which are discussed here.

\section{B. Mixing transmission platforms}

Clearly the presented setup is challenging, since coherent and non-coherent technologies are mixed together. Vendor $\mathrm{Z}$ uses a non-coherent transponder and accounting for dispersion via the dispersion non-compensated domain $(\mathrm{Y})$ is an operational issue. Two Dispersion Compensation Modules (DCMs) have been added (see Fig. 2). The dispersion within domain $\mathrm{X}$ was compensated via post-compensation, whereas the dispersion within domain $\mathrm{Y}$ was handled via the existing compensation modules. This challenge indicates clearly that AW deployment is a custom-engineering task. Depending on the properties of the WDM platform and the alien transponder at hand different parameters need to be controlled and taken into account. This calls for a more thorough framework, which will allow the planning for deployment of AWs to be as easy as for native services. ITU-T has already made first steps in this direction via their G.698.X standards, but these need to be updated for $40 \mathrm{G}$ (and above) data rates and possibly also for flex-grid applications in order to make the AW concept future-proof.

\section{Service activation procedures}

The WDM platforms in our test-bed had clearly two totally different approaches towards the support of AWs. Since native services are supported by a full set of OAM\&P functions, this requires the management systems to be "aware" of the services in the system. For both vendors this is handled by managing the services in the OCh layer of the OTN equipment allowing the optical signal to originate from alien transponders. However the mechanisms for provisioning the services and for ensuring the optical performance of the alien wavelengths differ significantly. Future systems will have more flexible and integrated management and control planes where service establishment will be automatic via a user-friendly interface. For this test-bed the service activation procedure required manual setup of the power levels and the interface points for both vendors. Nevertheless, the involved procedures were not that different from the native service setup procedure. In fact, an alien wavelength could be set up within 5 minutes. Thus, from operational point of view, the differences between the service activation procedures are minor. A potential drawback and a factor to be considered when calculating the CAPEX/OPEX of the AW solution is the required training of the personnel to set up such services.

Considering the systems under investigation, one of the main challenge in establishing the AW services was the adaptation of the power levels at the interfacing points - both at the Transponder-MUX sides and where the two domains interface via MUX-to-MUX inter-connection (see Fig. 2). Vendor X has explicit support for AW deployment and its automatic power equalization works for both native and alien signals. On the other hand, vendor Y does not have an explicit AW support 
and the automatic power regulation did not work for alien signals, i.e., the power levels of the AWs via domain Y had to be manually adjusted. This potentially error-prone procedure is an obvious drawback, but considering that the current trend is towards WDM platforms with intelligent automatic power control, such an issue is expected to be solved in the near future.

\section{Discussion}

The challenges we met during our experiments indicate that there is a clear need for more work, focused on establishing a proper framework for open WDM interface standard. It is clear that different vendors have different transmission system design approaches which while providing them with a unique solution, poses inter-operability challenges. The trade-off between competitiveness via proprietary engineering solutions and interoperability via open WDM interface is clear. First steps towards standardization are present in ITU-T's G.698.X standards, but the lack of commercial implementations indicates that the involvement of the WDM equipment vendors is paramount. While the AW solution might seem as unreasonable for the vendors, since they will loose revenue if the network operators do not buy their transponders, they could try and see themselves as the provider of the Alien transponder instead. Furthermore, opening up the WDM interface will boost innovation in the WDM layer (specifically the management and service planning functions) which potentially will provide new functionalities, flexibility and opportunities for new services.

\section{PERFormance Results}

Two experiments were conducted with the presented testbed. First, only the services from vendors $\mathrm{X}$ and $\mathrm{Y}$ were established and after manually adapting the power levels to operational values the services were left to run for four hours. Second, the third alien service from vendor $\mathrm{Z}$ was established and the impact of the establishment procedure on both native and existing alien signals was observed. Since the power adaptation in this integrated multi-domain environment is a challenge, it is expected that the new service establishment will potentially create disturbance on the existing services. For clarity of the presentation, the native services (10G), terminating at nodes B and C (see Fig. 2) are referred to as $10 \mathrm{G}$ far side. The $40 \mathrm{G}$ services have two sides an alien side, where the transponder is attached to the alien ROADM, and a native side, where the transponder is attached to a native ROADM.

\section{A. Experiment 1}

Fig. 3 presents the PreFEC BER readings over 4 hours with 15 mins. windowing period for the $40 \mathrm{G}$ services from both vendors $\mathrm{X}$ and $\mathrm{Y}^{3}$.

The difference in the PreFEC BER of the two types of $40 \mathrm{G}$ alien transponders is attributed to different implementations

\footnotetext{
${ }^{3}$ Vendor $\mathrm{Y}$ did not have measurement for the max PreFEC BER in the management system
}

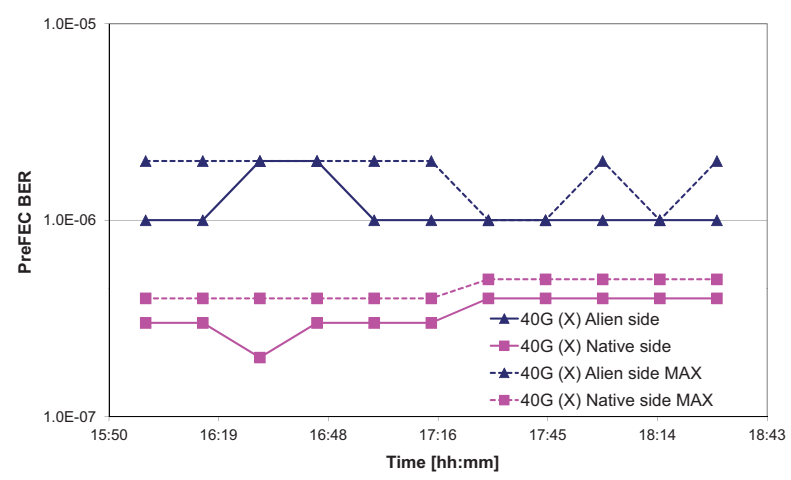

(a) PreFEC BER for the AW from Vendor $\mathrm{X}$.

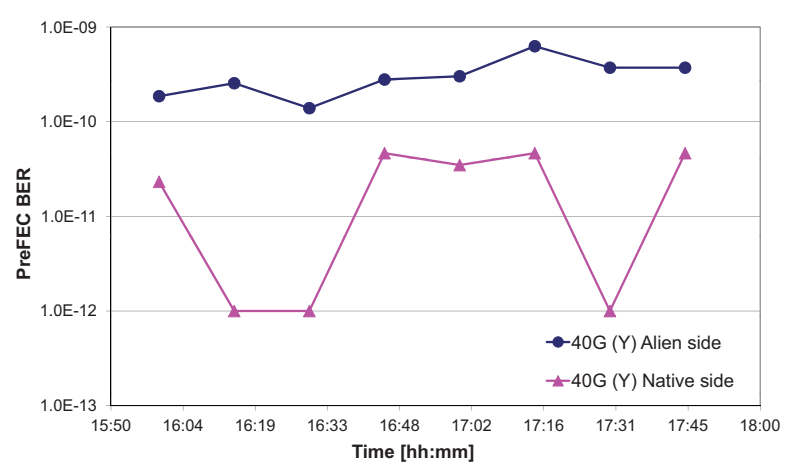

(b) PreFEC BER for the AW from Vendor Y.

Fig. 3. PreFEC BER performance of both $40 \mathrm{G}$ alien signals after 4 hours of service.

and the use of different coherent modulation formats (see Fig. 2). The FEC applied in both type of transponders is eliminating errors below PreFEC BER levels of $10^{-4}$. Thus, the observed BER levels are negligible from operational point of view. Furthermore, the difference between the PreFEC BER levels observed for the two transponders from vendor $\mathrm{Y}$ is of no practical significance for the service and may be attributed to sensitivity tolerances of the transponders. The OSNR of the native waves, measured on the ROADMs line receiver amplifiers, was better than $26 d B$ in each of the domains. This results in an estimated in-band OSNR of the alien waves of approximately $23 d B$, which is within standard operational margins.

\section{B. Experiment 2}

In this experiment the impact on existing (alien and native) services of establishing an AW service was observed. The PreFEC BER levels around the time of alien service creation for the $40 \mathrm{G}$ AWs are presented on Fig. 4(a). Considering that the applied FEC in both types of transponders eliminates errors below PreFEC BER levels of $10^{-4}$ it is clear that the observed service impact is negligible. No impact on the PreFEC BER for the $10 \mathrm{G}$ services was observed in either domain. Fig. 4(b) presents the received power levels for both $40 \mathrm{G}$ signals. The level of impact is minor, which also results in the minor effect on the preFEC BER levels. Fig. 4(c) presents the changes in the received power levels for the $10 \mathrm{G}$ services (far end 
side) for both vendor $\mathrm{X}$ and vendor $\mathrm{Y}$. The main impact is observed for the services from vendor $\mathrm{X}$, since this domain is the one applying automatic power regulation. It can be seen that the effects on the services from vendor $\mathrm{X}$ happen between 14:45 and 15:00, which is exactly when the service establishment in domain $\mathrm{X}$ was finalized and the automatic power regulation was initiated. At service decommissioning, similar minor effects were observed. The AW from vendor $\mathrm{Z}$ ran error-free throughout the entire experiment.

The presented results indicate that with this generation of WDM equipment even in a highly heterogeneous environment, by applying standard deployment procedures and by manually regulating the power levels of the alien services, the performance hit of the AW provisioning on existing services can be negligible. Note that only a minor performance impact of the alien wavelength provisioning was observed on the PreFEC BER of the two already provisioned alien wavelengths in the system. Nevertheless, care must be taken when multiple AWs from diverse vendors are accommodated in the network as the transponders might have different sensitivity and robustness to power changes in the system.

\section{CONCLUSION}

This paper presents a validation test-bed experiment for network expansion via open WDM interfaces, a.k.a Alien Wavelengths. We mixed coherent and non-coherent network segments and demonstrated establishment of two cross-domain $40 \mathrm{G}$ services and one $10 \mathrm{G}$ service, together with four $10 \mathrm{G}$ native services. No service disruption on either channel was observed. Furthermore, the procedures for provisioning the AWs have shown to be rather straightforward, similar to provisioning native services. The only encountered challenge was the power adaptation of the new services, which involves manual re-configurations and potentially will require additional training of the personnel for handling the service.

The presented experiment validates the feasibility of deploying AWs for metro-area network expansion purposes, which could potentially lower investment costs. Network operators can interconnect new network segments based on components from different vendors which opens up the possibilities for flexible network evolution and service deployment by using components with functionalities, which are not available from the vendor of the currently deployed WDM system. In this scenario, since both domains belong to the same network operator, monitoring, maintenance and alarm correlation could be achieved via proprietary management integration within the operators network. This opens up possibilities for flexible and rapid service delivery as well as for the deployment of an all-optical network paradigm.

\section{REFERENCES}

[1] O. Gerstel, R. Cassata, L. Paraschis, and W. Wakim, "Operational Solutions for an Open DWDM Layer," in OFC/NFOEC, March 2009.

[2] D. Ventorini, E. Moura, L. Paraschis, O. Gerstel, M. Silva, and K. Wollenweber, "Demonstration and Evaluation of IP-over-DWDM networking as "alien-wavelength" over existign carrier DWDM infrastructure," in OFC/NFOEC, Feb. 2008.

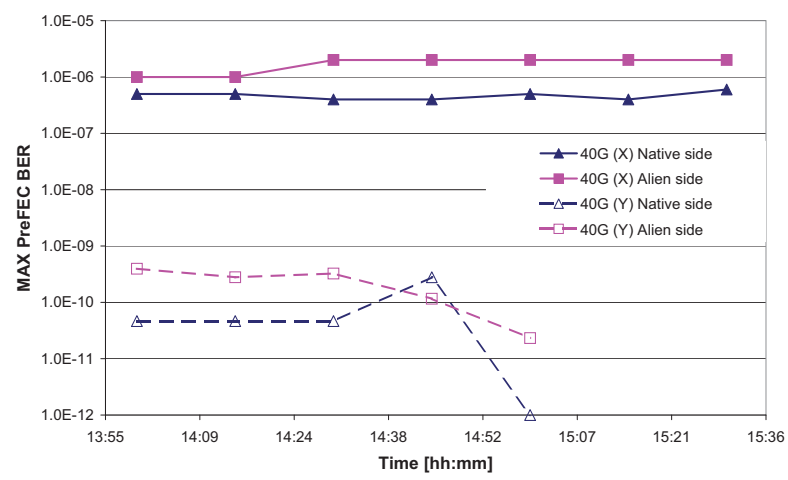

(a) PreFEC BER for the both $40 \mathrm{G}$ services.

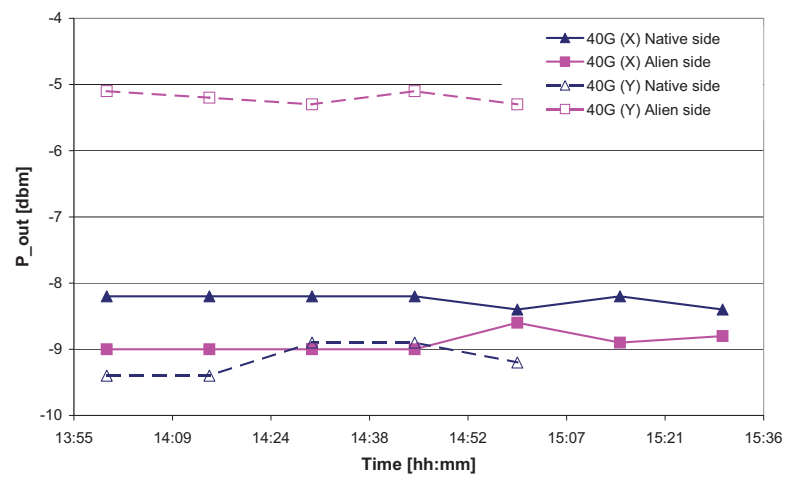

(b) Received power for both $40 \mathrm{G}$ services.

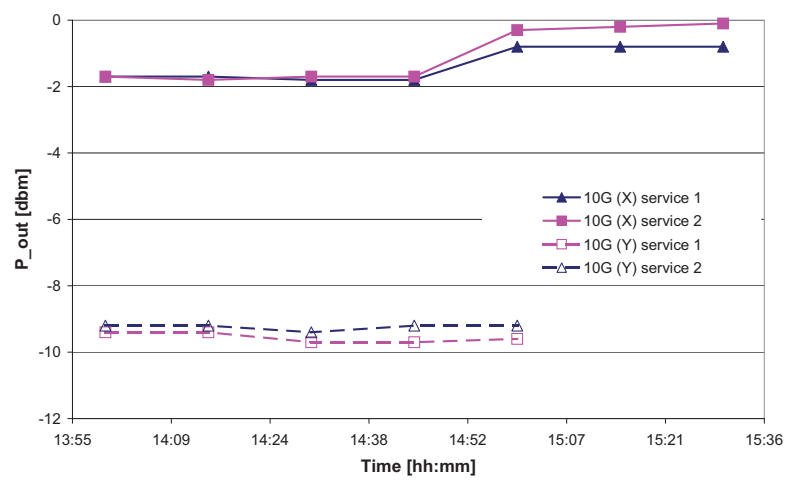

(c) Received power for the $10 \mathrm{G}$ services (far end).

Fig. 4. Service performance impact under AW establishment.

[3] A. Lord, Y. R. Zhou, P. Wright, P. Willis, C. Look, G. Jeon, S. Nathan, and A. Hotchkiss, "Managed Alien Wavelength Service Requirements and Demonstration," in ECOC, Sept. 2011.

[4] K. Slavicek and V. Novak, "Introduction of Alien Wavelength into CESNET DWDM Backbone," in 6th International Conference on Information, Communications \& Signal Processing, ICICS 2007, Dec. 2007.

[5] L. L. Bjørn, R. Nuijts, M. N. Petersen, and A. Manolova, "Design and OAM\&P aspects of a DWDM system equipped with a $40 \mathrm{~Gb} / \mathrm{s}$ PM-QPSK alien wavelength and adjacent $10 \mathrm{~Gb} / \mathrm{s}$ channels," in TERENA Networking Conference, TNC 2011, May 2011.

[6] A. Lord and C. Engineer, "OPEX savings for all-optical core networks," in $E C O C$, Sept. 2009.

[7] S. Melle, G. Bennett, C. Liou, C. Villamizar, and V. Vusirikala, "Alien Wavelength Transport: An Operational and Economic Analysis," in OFC/NFOEC, March 2009. 